Revision 2, 13 Jan 2009

\title{
A method to quantify alteration of knee kinematics caused by changes of TKR
}

\section{positioning}

\author{
Kessler $\mathrm{O}^{1}$,Bull $\mathrm{A} \mathrm{M} \mathrm{J}^{2}$, Amis, $\mathrm{A} \mathrm{A}^{3}$
}

Author details $\quad{ }^{1} \mathrm{O}$ Kessler MD, Stryker Orthopaedics, Florastr 13, CH 8800 Thalwil, Switzerland.

${ }^{2}$ AMJ Bull PhD, Reader in Musculoskeletal Mechanics, Department of Bioengineering, Imperial College London, London SW7 2AZ, United Kingdom

3 AA Amis DSc(Eng), Professor of Orthopaedic Biomechanics, Departments of Mechanical Engineering and Musculoskeletal Surgery, Imperial College London, London SW7 2AZ, United Kingdom

Corresponding Author:

Prof AA Amis DSc FIMechE

Mechanical Engineering Department

Imperial College, London SW7 2AZ, U.K.

Tel +44 $2075947062 /$ fax +44 2075947238

a.amis@imperial.ac.uk 


\section{ABSTRACT}

Few in vitro studies have investigated changes in kinematics caused by total knee replacement (TKR) implantation. The advent of surgical navigation systems allows implant position to be measured accurately and the effects of alteration of TKR position and alignment investigated. A test rig and protocol were developed to compare the kinematics of TKR-implanted knees for different femoral component positions. The TKR was implanted and the component positions documented using a navigation system. The quadriceps was tensed and the knees were flexed and extended manually. Torques and drawer forces were applied to the tibia during knee flexion-extension, while recording the kinematics with the navigation system. The implant was removed and replaced on an intramedullary fixation that allowed proximal-distal, and internal-external rotation of the femoral component without conducting a repeated arthrotomy on the knee. The implant was repositioned using the navigation system to reproduce the previously achieved normally-navigated position and the kinematics were recorded again. The recorded kinematics of the knee were not signficantly different between both normal implantation and intramedullary remounting for tibial internalexternal rotation, varus-valgus angulation, or posterior drawer, at any angle of knee flexion examined. Anterior drawer was increased approximately $2.5 \mathrm{~mm}$ across the range $20-35$ degrees knee flexion $(p<0.05)$, but was otherwise not significantly different. This method of navigating implant components and of moving them within the closed knee (thus avoiding artefactual effects of repeated soft tissue manipulations) can now be used to quantify the effect on kinematics of alteration of the position of the femoral component. (248 words) 


\section{INTRODUCTION}

Although there has been much work to refine the design of total knee replacement (TKR) components and their associated implantation procedures, there remains much scope for improving the functional outcome. The reasons for imperfect function are not completely understood, but it is known that the kinematics and stability of the replaced knees are often abnormal (Ulvehammer et al., 2000a,b; 2001).

The gross kinematics of the knee can be measured in a gait analysis laboratory, when the subject is usually filmed by a number of cameras (Sudhoff et al., 2006). However, this is not accurate enough to quantify the detailed kinematic behaviour of the knee. Greater accuracy is attained by methods such as fluoroscopy, but these are often in limited situations such as ascending a step (Banks et al., 1996; 1997; Kessler et al, 2007). There has been recent development of a mobile fluoroscopy unit, to track the knee of a walking subject (Zihlmann et al., 2006). While it is always best to obtain data for normal activities in-vivo, clinical and ethical considerations mean that it is usually difficult to set up strong prospective, randomised, comparative studies and, even then, there remain many uncontrolled variables relating to both the patient and the surgery. These include surgical factors or implant design. These factors mean that the effects of specific details, such as implant position, are difficult to discern. The recent introduction of computer-aided surgical navigation systems (Laskin and Beksac, 2006; Stockl et al., 2004) has now enabled TKR component positioning to be both controlled and documented accurately. This development means that it is now possible to study the effects of variation of implant position within the knee. In order to eliminate patient-related variables, intra-subject comparative studies may be done, but that entails working in-vitro, to allow re-positioning of TKR components.

It is known that small changes in the surgical procedure can cause significant functional effects after TKR. There have been studies on the effect of femoral component internal- 
external rotation and how that affects patellar tracking (Armstrong et al, 2003; Miller et al., 2001), for example. However, if studies are to be done to compare knee behaviour with different TKR positions, it is important that the process of moving the implant does not cause irreversible changes such as tearing of the soft tissues. This means that a method must be developed that allows the TKR to be moved in relation to the bones without repeatedly opening and closing the soft tissues.

The aims of this study were to design an experimental procedure to allow the positions of the components of a standard navigated TKR to be documented, then for those positions to be reproduced using an adjustable intramedullary mounting so that the component position can be altered without opening the knee. A final aim was to check that this method of removing the standard TKR from its original navigated position, followed by replacing it on the intramedullary mounting, would not alter the behaviour of the knee. This would be done by measuring the envelope of knee joint kinematics (Bull et al, 2002, Lucas et al, 2006) at both stages of the procedure.

\section{METHOD}

Eight adult fresh-frozen disarticulated lower limbs were obtained in conformity with local legal requirements. They had been separated at the hip, so included the femoral head but not the acetabulum, and also included the foot. All were left-sided, had normal alignment, and no evidence of gross arthritic changes, ligamentous instability or previous surgery. Navigation trackers (Stryker knee navigation system, Stryker Leibinger, Freiburg, Germany) were fixed securely to the outer cortices of the femora and tibiae $150 \mathrm{~mm}$ from the knee joint line. For each specimen the position of standard navigation reference points at the centre of the femoral head and ankle were recorded using the navigation system: the centre of the femoral head was located by moving the leg in circumduction within a fixed artificial acetabulum; the ankle was defined by digitizing the medial and lateral malleloi. The leg was divided $200 \mathrm{~mm}$ above and below the joint line and the proximal femur and foot/ankle 
complex discarded. The knee was mounted in a dedicated loading rig, with the transepicondylar axis aligned approximately to the flexion-extension axis of the rig, by clamping the proximal end of the femur. The rig allowed unconstrained tibial motion relative to the femur, apart from control of flexion-extension. The tibia hung free, allowing flexion of the knee $\left(0-120^{\circ}\right)$ by moving the femur and applying displacing loads to the quasi-static tibia (Grood et al., 1988). Any lack of alignment between the actual axis of flexion of the knee and that of the test rig was accommodated by small secondary movements of the tibia in relation to the test rig; these secondary movements did not affect the tibio-femoral kinematics recorded, which were measured directly from the relative motions of sensors mounted on the moving bones.

A Scorpio CR (Stryker Orthopaedics, Mahwah, NJ, USA) posterior cruciate retaining TKR was inserted to a classical, standardized, alignment (Dorr and Boiardo, 1986). The distal femur was cut perpendicular to the computer estimation of the mechanical axis in both coronal and sagittal planes, the resection was $0-1 \mathrm{~mm}$ deeper than the thickness of the distal part of the femoral component. The rotation of the femoral component was determined by using both epicondyles (Berger et al, 1993) and Whiteside's line (Whiteside and Arima, 1995). The final cuts were made after ensuring accurate AP positioning with the navigation. The final femoral component positioning will be referred to as the 'navigated position'. Two 3 $\mathrm{mm}$ diameter dimples had been spark eroded in the articular surface of the anterior flange of the femoral component, allowing a navigation tripod marker to be located reproducibly on the component (Figure 1). The relative position of this tripod to the navigation tracker fixed to the femur was measured using the navigation system, thus defining the "navigated position" of the femoral component. Two countersunk screw holes were also made through the anterior flange, for later mounting (see below). These implant modifications were convenient for us, but not necessary for the method; other means for locating the navigation tracker and mounting the implant could be devised. 
The tibia was cut perpendicular to its anatomical axis (Dorr and Boiardo, 1986). The resection level accommodated the thickness of the insert, and the rotational alignment was referenced using the PCL and medial $1 / 3^{\text {rd }}$ of the tibial tuberosity. The tibial component was cemented and the femoral component 'press-fitted'. The arthrotomy was closed as in clinical practice with No1 Vicryl (Ethicon, Somerville, NJ, USA) in the fascial layers, and 2/0 Vicryl in the fat and 4/0 Vicryl for the skin.

To simulate the extensor mechanism a tension of $400 \mathrm{~N}$ was applied to the patella via a cable attached to a pneumatic cylinder parallel to the femoral axis (Figure 2). Tibial internalexternal rotation torques of $5 \mathrm{Nm}$ were applied by hanging weights on two cords connected to a disc attached to the distal end of an intramedullary rod inserted into the distal end of the tibia. These were applied in opposite medio-lateral directions to ensure no resultant forces were induced. Similary, tibial varus/valgus moments of $3.5 \mathrm{Nm}$ were applied using a cord/pulley system attached to the distal end of the intramedullary rod. Anterior-posterior (AP) drawer forces of $70 \mathrm{~N}$ were applied to the proximal tibia via a low friction bearing on a hoop, so that the resultant force was always through the centre of the tibial plateau and secondary rotations were not inhibited. The femur was then extended and flexed manually by lifting the femoral mounting, the $400 \mathrm{~N}$ extensor mechanism tension being insufficient to lift the weight of the test rig unaided. Each cycle took approximately 5 seconds (Figure 2).

Due to physical constraints of the application of posterior forces, only movements between 0-approximately $100^{\circ}$ were achievable. The navigation system was used to record the movement of the femur and tibia during the active knee extension motion from 90 to $0^{\circ}$ for the following loading conditions:

Internal rotation torque $(5 \mathrm{Nm})$, external rotation torque $(5 \mathrm{Nm})$, anterior drawer $(70 \mathrm{~N})$, posterior drawer $(70 \mathrm{~N})$, varus moment $(3.5 \mathrm{Nm})$, valgus moment $(3.5 \mathrm{Nm})$, and neutral (no additional loading other than the simulated extensor load). 
Kinematics data were described with six degrees-of-freedom. This consisted of a threecylinder open chain mechanism (Grood and Suntay, 1983) where flexion-extension was a tibial rotation about the digitised femoral epicondylar axis (Berger et al., 1993); internalexternal rotation was about the long axis of the tibia, the zero point of which was defined by the relative position of the malleolar mediolateral axis and the femoral epicondylar axis when the knee was fully extended; varus-valgus rotation was about a 'floating' axis mutually perpendicular to the other two axes, in the AP direction (Grood and Suntay, 1983). Translations were measured along these axes; only AP translation will be presented. The centre of the femoral head was known by sphere fit measurement; the centre of the knee was defined as the highest point of the anterior-distal outlet of the intercondylar notch; the centre of the ankle was the mid-point of the line joining the malleoli. These points were all digitised and defined by the navigation system, while the leg was intact.

On completion of these kinematic tests the knee was opened and the femoral component of the TKR was removed and the femoral intramedulllary (IM) cavity drilled and reamed along its length from the cut proximal end to approximately $50 \mathrm{~mm}$ proximal to the most proximal margin of the posterior femoral condyles. Two further cement keying holes were drilled bicortically across the femur. A brass tube was cemented with polymethylmethacrylate in the IM cavity (Figure 3). This had dimensions of $16 \mathrm{~mm}$ external diameter, $10 \mathrm{~mm}$ internal diameter and $250 \mathrm{~mm}$ in length. This brass tube included a milled flat on its external surface so that, combined with the cement interlocking with the bicortical holes in the femur, it would be able to resist torsional loads. A clamping mechanism was welded to the proximal end of the brass tube, that allowed a $10 \mathrm{~mm}$ stainless steel IM rod to be fixed rigidly within the brass tube inside the femur.

A series of steel rods were manufactured with varus offsets of 3,6 , and $9^{\circ}$ that allowed the femoral TKR components to be fixed rigidly to the rods in varying varus angulations (Figure 4). Slots in an intermediate mounting plate allowed medial-lateral adjustment. A set of intermediate plates were made and machined with different proximal-distal tapering 
thickness; this allowed flexion adjustment of the component to allow for different anterior bowing of the femur. The flat intermediate plate fitted against the anterior flange of the implant and did not locate onto specific features within the implant shell; thus the plate could be used with a range of TKR sizes. Anterior position was adjusted by placing spacer washers between the plate and the femoral component. When these features were combined with axial translation and internal-external rotation between the IM rod and the tube, this set-up allowed the femoral component position to be adjusted in all 6 degrees-offreedom.

Additional cuts were made to trim the distal femur that would allow for future internal-external rotation and proximal-distal repositioning of the femoral component using the IM fixation. Before fixing the component, tests were made to ensure that the component could be rotated about the axis of the IM rod (i.e. internal/external rotation) by $\pm 10^{\circ}$. Further cuts of the distal femoral condyles were made if necessary to allow this range of femoral component rotation. The femoral component was repositioned to the original navigated position using the navigation tripod located on the femoral component (Figure 1). This was confirmed within $1 \mathrm{~mm}$ and $1^{\circ}$ of the original position in all three directions (referred to as the 'IM position').

After "navigating" the femoral component back to the original reference position, it was fixed in place using the brass locking mechanism. The soft tissues were closed as before and the kinematics measurements for all loading conditions were then repeated.

Paired two-way t-tests were used to test the hypothesis that there was no difference in the kinematics of the navigated knee to the IM positioned knee with an alpha level of 0.05 . The independent variable was knee joint flexion-extension, the dependent variables were the primary motions of the knee, i.e. AP position, tibial internal-external rotation, and tibial varus/valgus angulation. Coupled motions were not analysed. The average of three extension cycles over the range $90-0^{\circ}$ was used for analysis. Retrospective power analyses showed that, for $n=8$ knees, a difference of anterior translation of $1.5 \mathrm{~mm}$, posterior 
translation $1.6 \mathrm{~mm}$, internal rotation of $1.9^{\circ}$, external rotation $2.1^{\circ}$, varus and valgus rotations of $1.6^{\circ}$ could be identified with $80 \%$ power with $95 \%$ confidence.

\section{RESULTS}

Kinematics data are presented in paired graphs where all navigated component TKR data are presented as a mean \pm standard deviation from the mean. When examining the difference between navigated femoral position and IM position the $95 \%$ confidence interval (Cl) bands of this difference are plotted at each degree of knee flexion. Thus, according to the sign test, a statistically significant difference is indicated when the $95 \% \mathrm{Cl}$ bands for the IM position do not overlap the mean line for the navigated position.

The tibial internal-external rotation envelope of laxity for the navigated knee is shown in Figure 5a and for the IM position of the femoral component in Figure $5 \mathrm{~b}$. The data does not show a statistically significant difference in the internal-external rotation envelope of laxity across the range of knee flexion examined.

Figure 6 shows similar data for varus-valgus laxity. Again, no statistically significant change is shown between the navigated TKR and after re-mounting the femoral component on the IM rod.

Figure 7 shows that, while the envelope of tibial posterior drawer laxity did not differ significantly between the navigated and IM states, the tibial anterior drawer had decreased by approximately $2 \mathrm{~mm}(\mathrm{p}<0.05)$ across the arc from 35 to $26^{\circ}$ knee extension.

\section{DISCUSSION}

The aim of the study was to develop an experimental set up that allows the precise recording of knee kinematics on cadaver knee specimens while changing loading conditions. This paper has presented an additional technique that allows the position of a navigated TKR implant to be documented in relation to the bone on which it is mounted, then for the prosthesis to be removed from the body and subsequently navigated back into the same 
position again, after it has been re-mounted onto an adjustable fixture. In the example illustrated, this fixture allowed the femoral component position to be altered after the arthrotomy of the knee joint had been closed, enabling study of the effects of femoral component internal-external rotation, or changes of joint line height, without subsequent disturbance of the soft tissues. The results show that the kinematics and envelopes of laxity of knee extension were not affected significantly by removing and re-positioning the prosthesis, apart from a small decrease in anterior translation laxity that was found over a short flexion arc. We do not have an explanation for the decrease in anterior laxity - the work done on the knee was expected, if anything, to cause increased laxity, due to capsular damage, for example. The only other mechanism that we speculate is that there may have been overtightening of the capsular structures when the joints were resutured.

The clinical literature includes many studies that have shown how small changes in the positions of knee prostheses have large effects on the function of the knee. In particular, some designs of TKR and associated instruments build-in a 3 degrees external rotation of the femoral component, which is intended to optimize factors such as patellar tracking (Berger et al., 1998). Work intended to study the effects of similar changes in TKR position has been limited by inability to place TKR components consistently, to be able to move them to new positions accurately, or to do this without causing additional changes by the surgical procedure in the surrounding soft tissues that may influence knee kinematics (for example, ligament release). The experimental design presented here included the use of an optical navigation system that has allowed all these problems to be overcome. In addition, by attaching a navigation tracking target to the protruding end of the IM rod, in addition to one secured to the femur, it is possible to change the position of the femoral component by known amounts while the knee joint remains closed. This means that changes in knee behaviour will be caused solely by the change in position of the implant and unaffected by repeated opening and re-suturing of the soft tissues, a process that could be expected to cause other unwanted effects. This method could be applied to other joints, such as the 
elbow, and could also be used in studies of revision TKR in which substantial bone loss makes alignment and implant positioning difficult. The method could also be applied to studies where the joint is mounted in different types of test rig.

Although data obtained from patients in-vivo is always preferable, the inherent variability of factors such as individual gait patterns, surgical influence, or component alignment means that it may not be possible to obtain adequate statistical power to identify small changes in joint kinematics, even with large numbers of subjects. The strength of this work in-vitro is that factors such as joint loading can be standardized and controlled carefully. Furthermore, it then becomes possible to examine several TKR set-ups in each knee. This eliminates variability of factors such as individual ligament properties and allows repeated-measures statistical analysis of the kinematic data.

The testing method described, in which the femur was flexed and extended while the tibia remained approximately stationary, allowed the collection of kinematic data that described "envelopes of laxity". It also simplified the task of applying a constant displacing load to the tibia, across the range of knee flexion: by keeping the tibia vertical, this eliminated changing gravitational effects. This set-up was pioneered for whole-leg specimens by Grood et al (1988), although they did not apply the quadriceps tension, that is necessary to maintain compression of the tibio-femoral joint. A further development has been to calculate the $95 \%$ confidence intervals at every degree of knee flexion: this allows the exact arc of motion, over which a statistically significant change occurs (if any), to be identified.

\section{Conclusions:}

This paper has described a method that allows the position and orientation of joint prosthesis components to be measured, after which they can be removed from a human joint, then setup on an adjustable mounting and navigated back to their datum position. The adjustable mounting then allows the prosthetic components to be navigated to different positions within the closed joint, so that multiple intra-specimen comparative tests of the effects of different 
implant positions can be made. This should be a powerful method for identifying how small differences in the set-up of a joint replacement affect the joint kinematics, so that these variables may be matched to normal knee kinematics.

\section{Acknowledgements}

This study was funded by Stryker (Europe) SA. This paper is based on experimental work that was carried out primarily by Mr Duncan Lucas, but the calculations, analysis and discussion are those of the authors alone. We thank Mr Philip Wilson for his work in making the test rig. The Clinical Research Fellowship for Mr D. Lucas was provided by $\mathrm{Mr} \mathrm{S}$. Chauhan of Brighton University Hospital from funds provided by Stryker (UK) Ltd. 


\section{References}

Armstrong, A.D., Brien, H.J., Dunning, C.E., King, G.J., Johnson, J.A., Chess, D.G. , 2003. Patellar position after total knee arthroplasty: influence of femoral component malposition. J Arthroplasty 18, 458-465.

Banks, S.A., Hodge, W.A., 1996. Accurate measurement of Three-Dimensional Knee Replacement Kinematics using Single-Plane Fluoroscopy. IEEE Trans Biomed Engng $43,638-649$.

Banks, S.A., Markovich, G.D., Hodge, W.A., 1997. In-vivo kinematics of cruciate-retaining and substituting knee arthroplaties. J Arthrop 12, 297-304.

Berger, R.A., Crossett, L.S., Jacobs, J.J., Rubash, H.E., 1998. Malrotation causing patellofemoral complications after total knee arthroplasty. Clin Orthop 356, 144-153.

Berger, R.A., Rubash, H.E, Seel, M.J., Thompson, W.H., Crossett, L.S., 1993. Determining the rotational alignment of the femoral component in total knee arthroplasty using the epicondylar axis. Clin Orthop 286, 40-47.

Bull, A.M.J., Earnshaw, P.H., Smith, A., Katchburian, M.V., Hassan, A.N.A., Amis, A.A., 2002. Intraoperative measurement of knee kinematics in reconstruction of the anterior cruciate ligament. J. Bone Joint Surg [Br]. 84-B, 1075-1081.

Dorr, L.D., Boiardo, R.A., 1986. Technical considerations in total knee arthroplasty. Clin Orthop Relat Res. 205, 5-11.

Grood, E.S., Stowers, S.F., Noyes, F.R., 1988. Limits of movement in the human knee. Effect of sectioning the posterior cruciate ligament and posterolateral structures. $\mathrm{J}$ Bone Jt Surg [Am] 70-A, 88-97.

Grood, E.S., Suntay, W.J., 1983. A joint coordinate system for the clinical description of Three-Dimensional motions: Application to the knee. Trans ASME J Biomed Engng $105,133-144$.

Hsu, H.P., Garg, A., Walker, P.S., Spector, M., Ewald, F.C., 1989. Effect of knee component alignment on tibial load distribution with clinical correlation. Clin Orthop 248, 135-144. 
Kessler, O., Durselen, L., Banks, S., Mannel, H., Marin, F., 2007. Sagittal curvature of total knee replacements predicts in vivo kinematics. Clin Biomech 22, 52-58.

Laskin, R.S., Beksac, B., 2006. Computer-assisted navigation in TKA: where we are and where we are going. Clin Orthop Relat Res 452, 127-131

Lucas, D.J., Alam, M., Bull, A.M.J., Kessler, O., Amis, A.A., 2006. Effect of internal-external rotation position of the femoral component on kinematics of the knee post TKR. J Bone Joint Surg [Br] Proceedings 88-B: 444.

Miller, M.C., Berger, R.A., Petrella, A.J., Karmas, A., Rubash, H.E., 2001. Optimizing femoral component rotation in total knee arthroplasty. Clin Orthop Relat Res. 392, 38-45.

Stockl, B., Nogler, M., Rosiek, R., Fischer, M., Krismer, M., Kessler, O., 2004. Navigation improves accuracy of rotational alignment in total knee arthroplasty. Clin Orthop Relat Res. 426, 180-186.

Sudhoff, I., Van Driessche, S., Laporte, S., de Guise, J.A., Skalli, W., 2007 Comparing three attachment systems used to determine knee kinematics during gait. Gait Posture 25, $533-543$.

Uvehammer, J., Kärrholm, J., Brandsson, S., Herberts, P., Carlsson, L., Karlsson, J., Regnér, L., 2000. In-vivo kinematics of Total Knee Arthroplasty: Flat compared with concave tibial joint surfaces. J Orthop Res 18 , 856-864.

Uvehammer, J., Kärrholm, J., Brandsson, S., 2000. In vivo kinematics of total knee arthroplasty. Concave versus posterior-stabilised tibial joint surface. J Bone Joint Surg [Br] 82-B, 499-505.

Uvehammer, J., 2001. Knee Joint kinematics, fixation and function related to joint area design in total knee arthroplasty. Thesis.Acta Orthop Scand suppl. 72, 1-52.

Whiteside, L.A., Arima, J., 1995. The anteroposterior axis for femoral rotational alignment in valgus total knee arthroplasty. Clin Orthop Relat Res 321, 168-172.

Zihlmann, M.S., Gerber, H., Stacoff, A., Burckhardt, K., Szekely, G., Stussi, E., 2006. Threedimensional kinematics and kinetics of total knee arthroplasty during level walking 
using single plane video-fluoroscopy and force plates: a pilot study. Gait Posture 24, $475-481$. 


\section{Figure Legends}

Figure 1. Navigation tripod on femoral component

Figure 2. Schematic and photograph of the experimental setup to measure the envelope of kinematics.

Figure 3. Intramedullary rod-in-tube mechanism to adjust joint line and femoral component rotation.

Figure 4. Distal mechanism to fix the femoral component to the IM rod in varying varus or flexion angulations and anterior or lateral offsets.

Figure 5. (a) Tibial internal-external envelope of laxity for the knee with the standard navigated TKR. The upper and lower lines are the mean limits of tibial external and internal rotation, respectively, caused by $+/-5 \mathrm{Nm}$ torque $(n=8$, mean+/-SD). (b) The change in tibial internal-external envelope of laxity after IM TKR positioning. - - - : mean position after standard TKR, from graph above; -------: mean position after TKR had been remounted on the intramedullary (IM) apparatus. $(n=8$, mean $\pm 95 \% \mathrm{Cl}$ of the difference between navigated TKR and intramedullary mounted TKR, no significant differences).

Figure 6. (a) Tibial varus-valgus rotation envelope of laxity for the knee with the standard navigated TKR. The upper and lower lines are the mean limits of tibial valgus and varus angulation, respectively, caused by $+/-3.5 \mathrm{Nm}$ moment $(\mathrm{n}=8$, mean+/-SD). (b) The change in tibial varus-valgus rotation envelope after IM TKR positioning. - - - : mean position after standard TKR, from graph above; -------: mean position after TKR had been remounted on the intramedullary (IM) apparatus $(n=8$, mean $\pm 95 \% \mathrm{Cl}$ of the difference between navigated TKR and intramedullary mounted TKR, no significant differences).

Figure 7. (a) Tibial posterior-anterior envelope of laxity for the navigated knee with the standard navigated TKR. The upper and lower lines are the mean limits of tibial anterior and posterior translation, respectively, caused by $+/-70 \mathrm{~N}$ drawer force $(\mathrm{n}=8$, mean+/-SD). (b) The change in tibial posterior-anterior envelope after IM TKR positioning. - - - : mean position after standard TKR, from graph above; -------: mean position after TKR had been remounted on the intramedullary (IM) apparatus $(n=8$, mean $\pm 95 \% \mathrm{Cl}$ of the difference 
between navigated TKR and intramedullary mounted TKR). There was a significant change in anterior laxity from 35 to $26^{\circ}$ knee extension. 
Figure 1 Navigation tripod on femoral component.

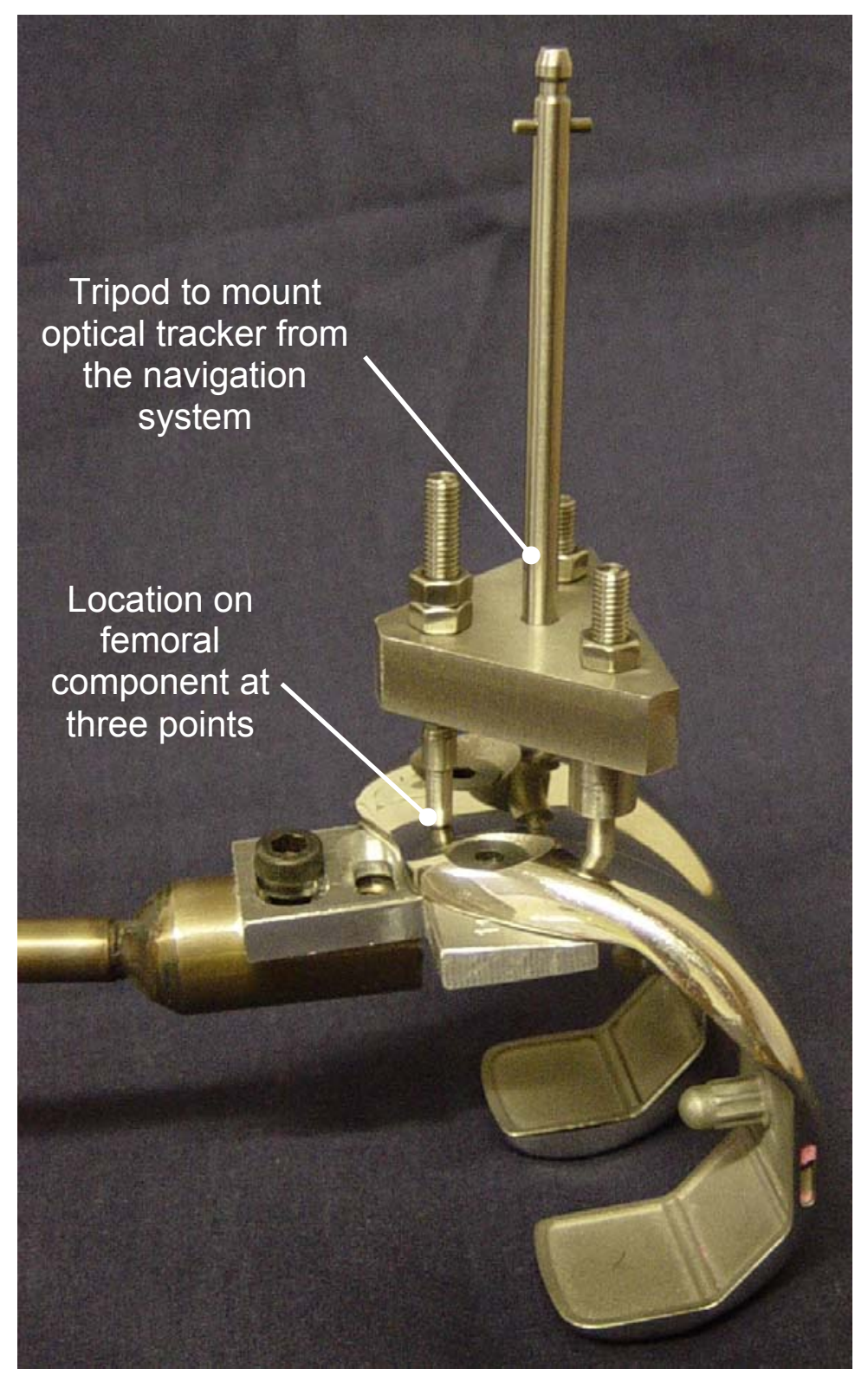


Figure 2 Schematic and photograph of the experimental setup to measure the envelope of kinematics.
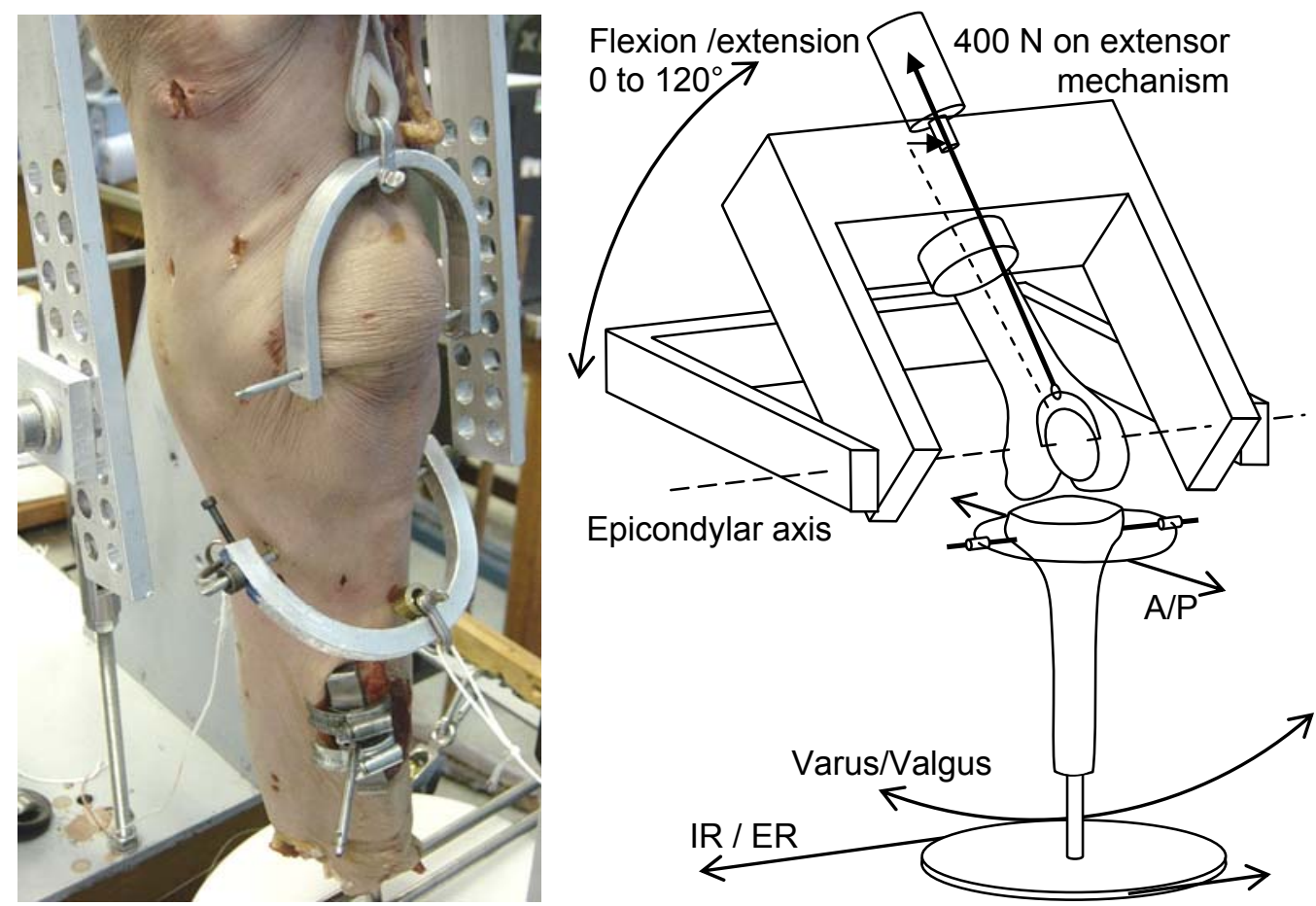
Figure 3 Intramedullary rod-in-tube mechanism to adjust joint line and femoral component rotation.

\begin{tabular}{|c|c|c|}
\hline $\begin{array}{c}\text { Stainless steel } 10 \mathrm{~mm} \\
\text { inner rod }\end{array}$ & $\begin{array}{c}16 \mathrm{~mm} \text { brass tube for } \\
\text { fixation in the femoral } \\
\text { intramedullary cavity }\end{array}$ & $\begin{array}{c}\text { Locking mechanism } \\
\text { allowing } 10 \mathrm{~mm} \text { inner rod } \\
\text { to be fixed rigidly to brass } \\
\text { tube }\end{array}$ \\
\hline $\begin{array}{c}\text { Femoral component } \\
\text { mounting with fixed valgus } \\
\text { angulation }\end{array}$ & $\begin{array}{c}\text { Allows femoral component } \\
\text { internal-external rotation } \\
\text { and alteration of joint line } \\
\text { height }\end{array}$ & $\begin{array}{c}\text { Location for navigation } \\
\text { tracker }\end{array}$ \\
\hline
\end{tabular}


Figure 4 Distal mechanism to fix the femoral component to the IM rod in varying varus or flexion angulations and anterior or lateral offsets.

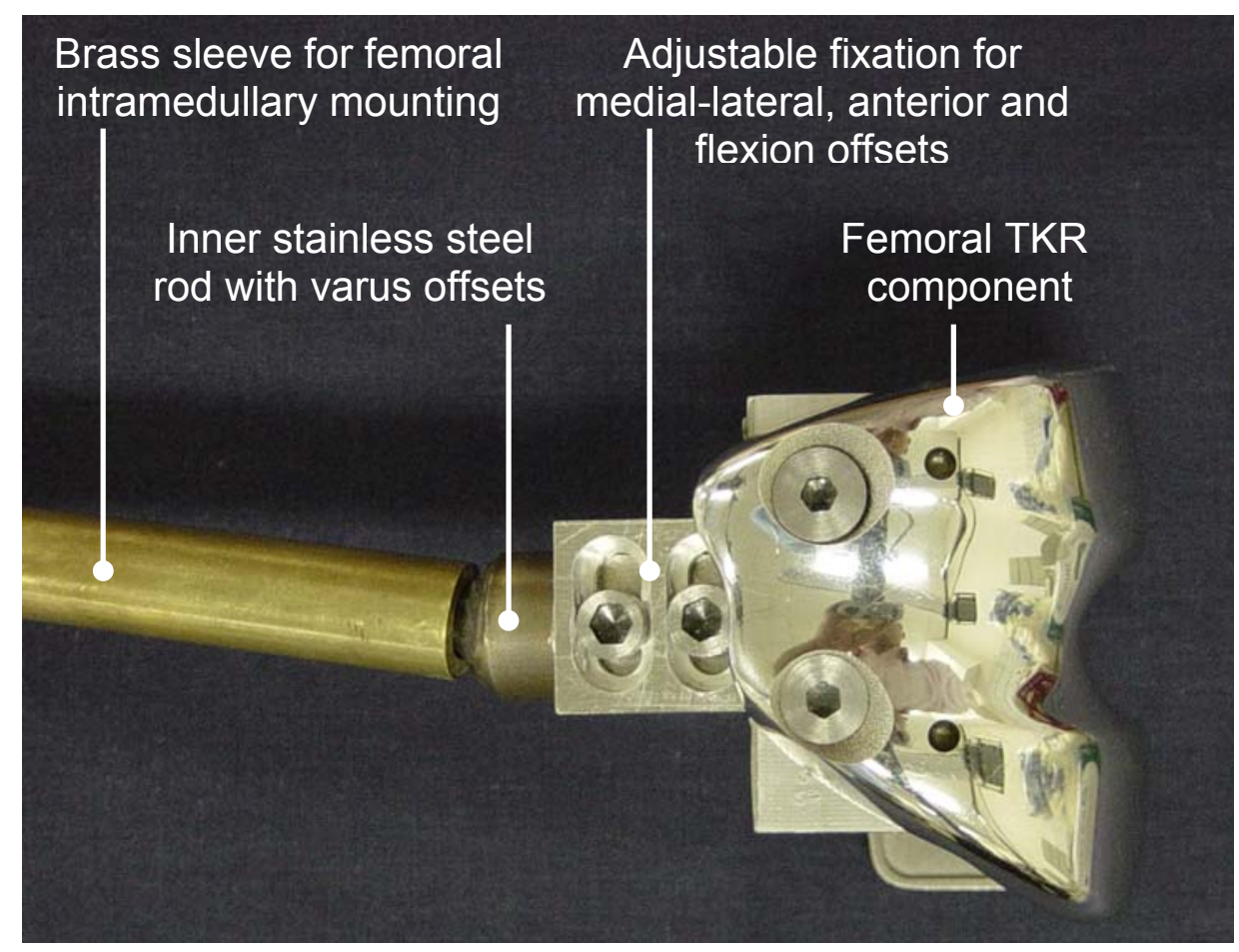


Figure 5a

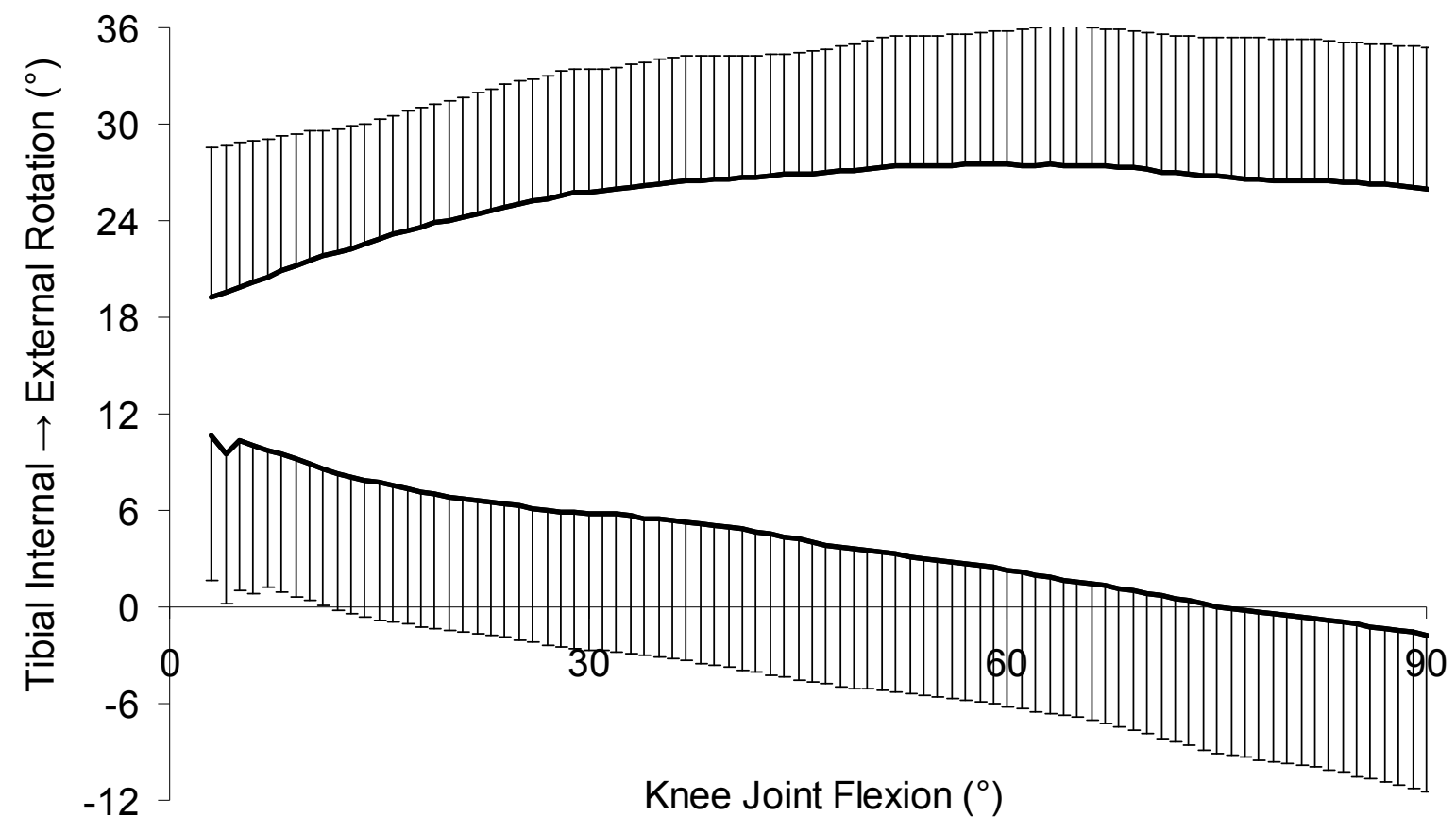

Figure 5b:

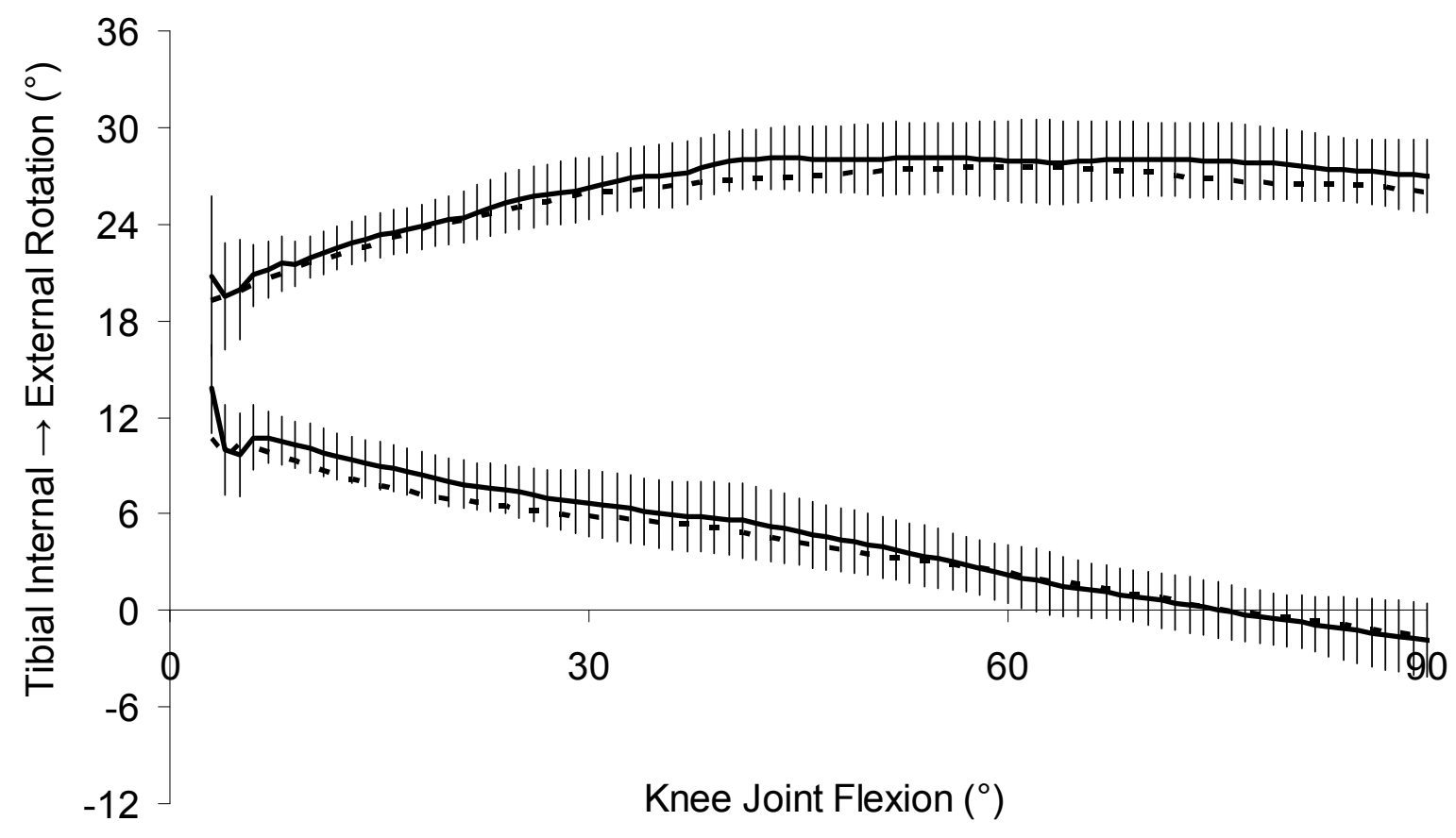


Figure 6a

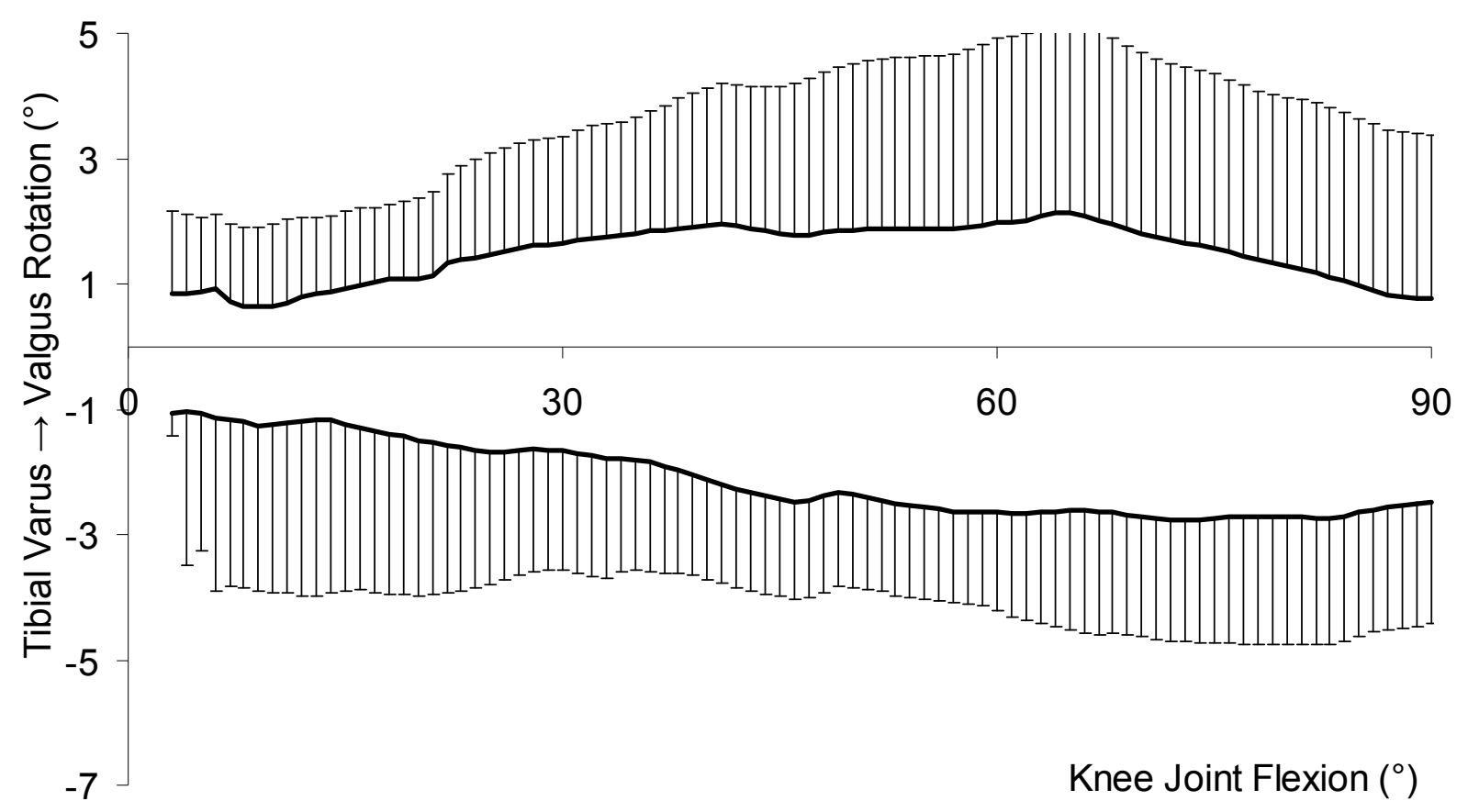

Figure 6b:

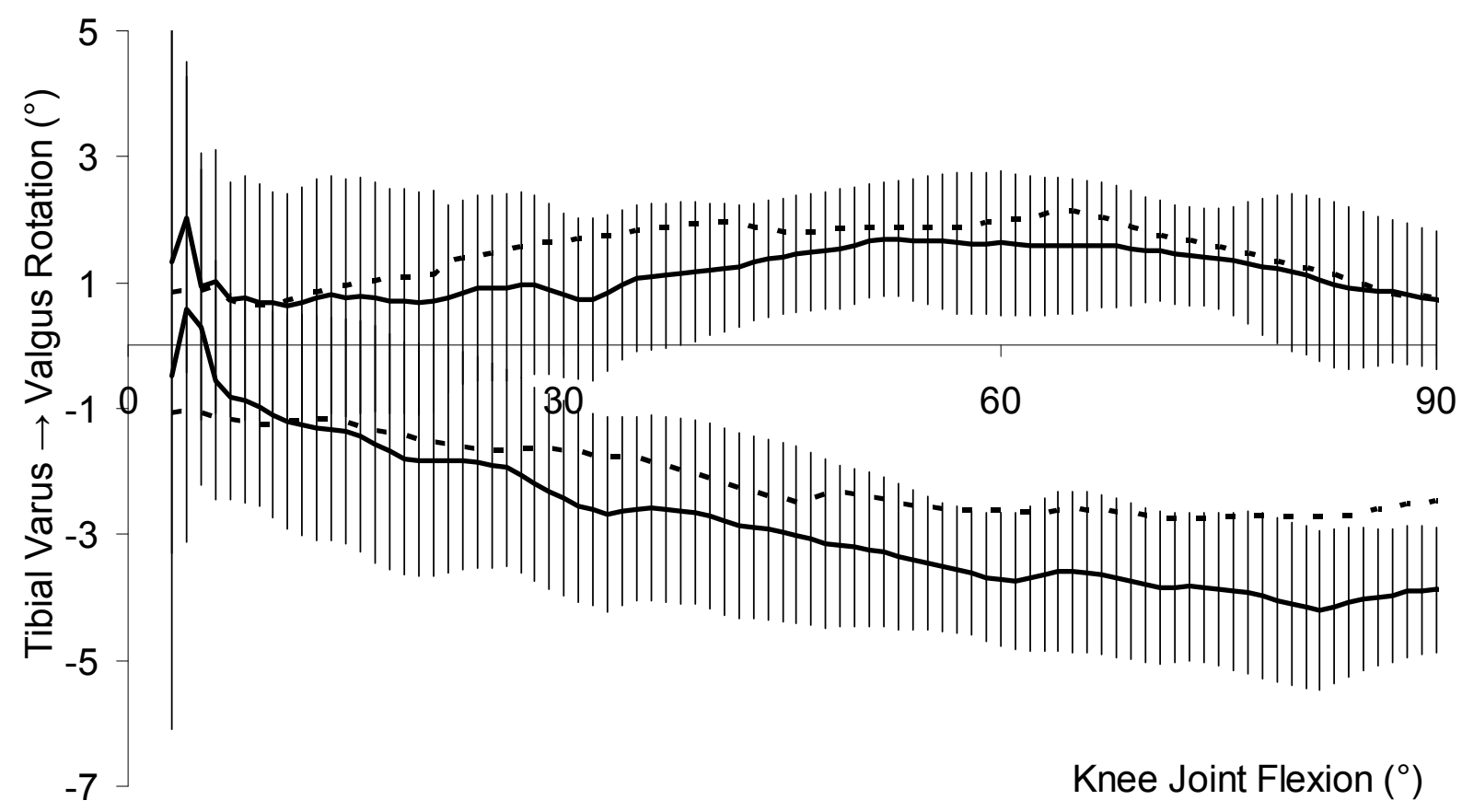


Figure 7a

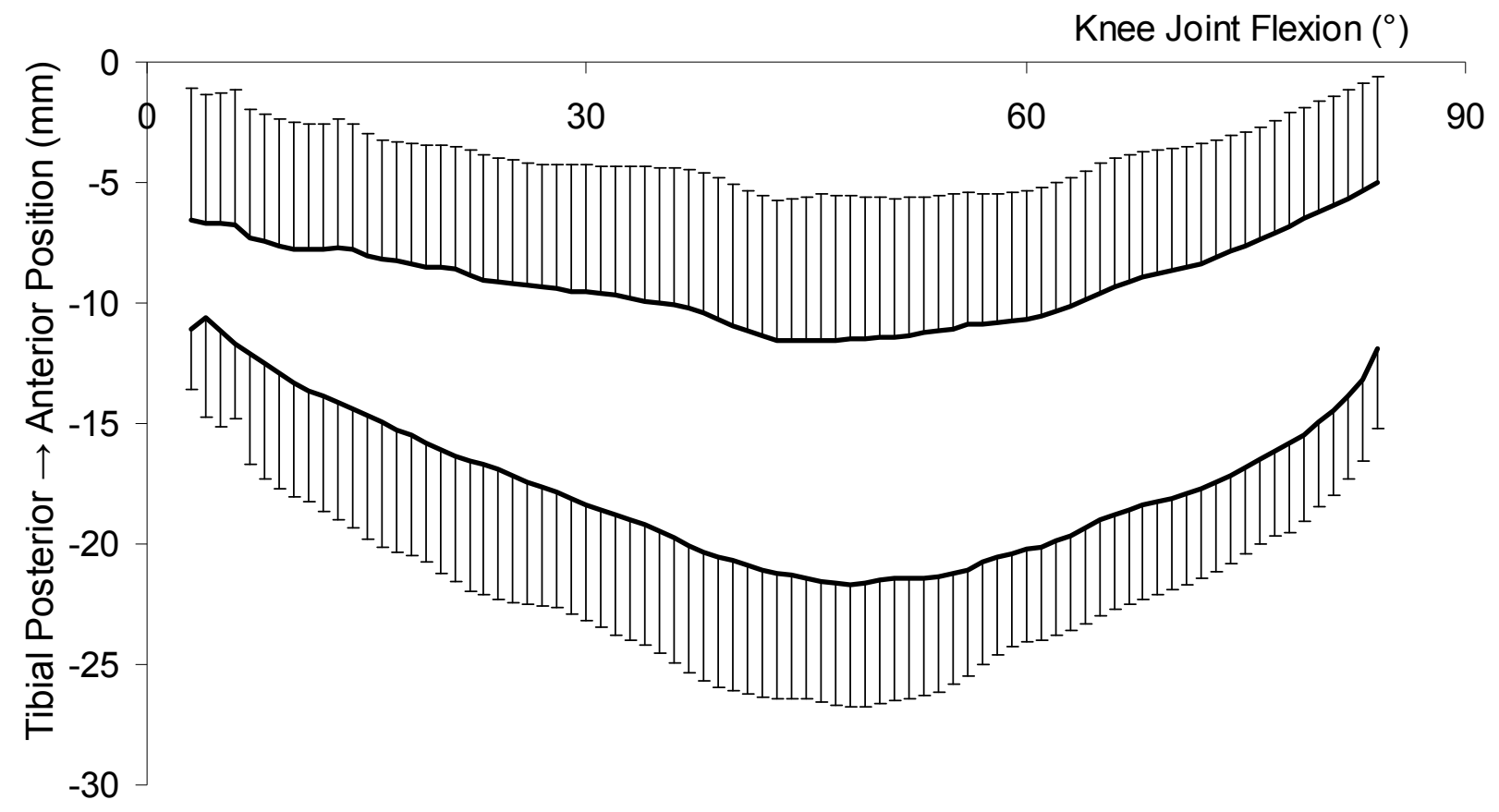

Figure 7b:

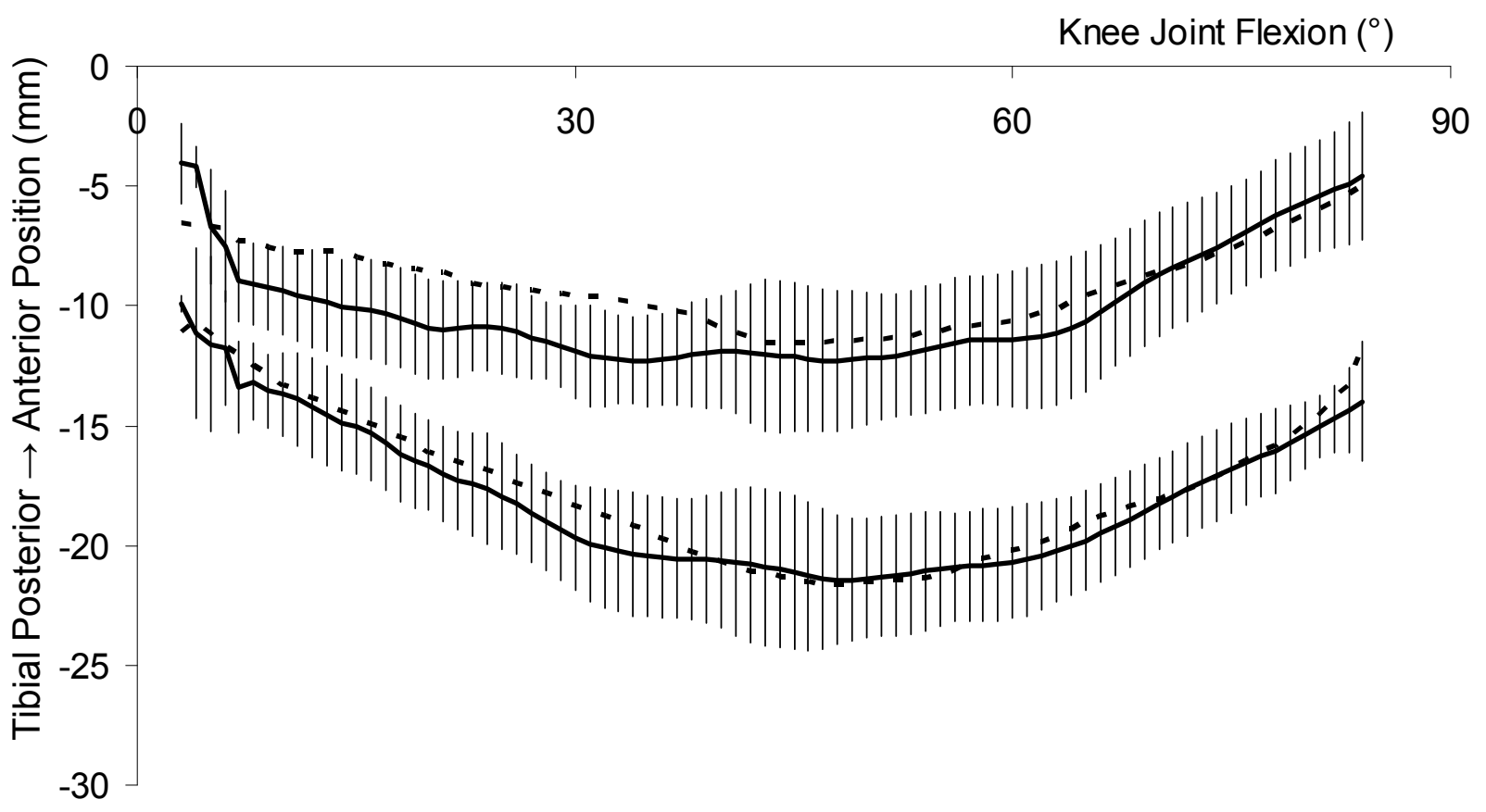

